\title{
Abnormal Savda Munziq, an Herbal Preparation of Traditional Uighur Medicine, May Prevent 1,2-dimethylhydrazine-Induced Rat Colon Carcinogenesis
}

\author{
Abdiryim Yusup, ${ }^{1}$ Halmurat Upur, ${ }^{1}$ Anwar Umar, ${ }^{1}$ Benedicte Berke, ${ }^{1,2}$ Dilxat Yimit, ${ }^{1}$ \\ Jaya Conser Lapham, ${ }^{1}$ Nicholas Moore, ${ }^{1,2}$ and Pierrette Cassand ${ }^{3}$ \\ ${ }^{1}$ Faculty of Traditional Uighur Medicine, Xinjiang Medical University, 830011 Urumqi, China \\ ${ }^{2}$ Department of Pharmacognosy, University Victor Segalen Bordeaux 2, 33076 Bordeaux, France \\ ${ }^{3}$ Laboratory of Food and Colon Carcinogenesis, University Bordeaux 1, 33405 Talence, France
}

Correspondence should be addressed to Halmurat Upur, halmurat@263.net

Received 3 July 2008; Accepted 12 May 2009

Copyright (C) 2011 Abdiryim Yusup et al. This is an open access article distributed under the Creative Commons Attribution License, which permits unrestricted use, distribution, and reproduction in any medium, provided the original work is properly cited.

\begin{abstract}
The study tried to assess the chemoprotective effect of abnormal Savda Munziq (ASMq) on 1,2-dimethylhydrazine (DMH)induced rat colon carcinogenesis. Male F344 rats were randomized into eight groups: Group 1 was served as control, no DMH injection was given and treated daily with normal saline. Rats in Groups 2-8 were given a single intraperitoneal injection of DMH $(20 \mathrm{mg} / \mathrm{kg}$ body weight) at the beginning of the study. Group 2 was served as negative control, administered with normal saline until the end of the experiment after the single DMH injection. Groups 3-5 were served as pretreatment group, administered with ASMq ethanol extract at 400, 800 and $1600 \mathrm{mg} / \mathrm{kg}$ body weight, respectively, until the 45th day, continued by normal saline administration for another 45 days. Groups 6-8 were served as the treatment group, administered with normal saline for the first 45 days from the day of DMH injection, ASMq ethanol extract at three different doses to be administered until the end of the second 45th day. All rats were sacrificed at 91 st day and the colons were analyzed for aberrant crypt foci (ACF) formation and crypt multiplicity. Results showed that ASMq ethanol extract reduced the number of ACF, AC and crypt multiplicity significantly $(P<$ $.05)$. It suggested that ASMq ethanol extract had chemoprotective effects on DMH-induced colon carcinogenesis, by suppressing the development of preneoplastic lesions, and probably exerted protection against the initiation and promotion steps of colon carcinogenesis.
\end{abstract}

\section{Introduction}

Abnormal Savda Munziq (ASMq), one of the Uighur medicinal herbal preparations, is widely distributed in the Xinjiang region of China. It has long been used in Traditional Uighur Medicine for the treatment of several diseases such as digestive cancer, diabetes, cardiovascular diseases or chronic asthma [1]. It is regularly used by Uighur physicians for the treatment of cancer, and in southern Xinjiang many ordinary people regularly self-medicate with it to prevent cancers. To date, these putative anticancer effects of ASMq have been little studied and its potential mechanism of action is still unclear. A possible anticancer effect of ASMq clearly warrants further investigation. In our previous studies, ASMq was proved to possess strong free radical scavenging effects, and decreased biological markers of oxidative stress in man [1], protected mitochondria and DNA against $\mathrm{OH}-$ induced oxidative damage in a cell-free system $[2,3]$, inhibited cancer cells proliferation and viability in vitro [4].

Colon cancer is one of the most common malignancies in many regions of the world and is thought to arise from the accumulation of mutations in a single epithelial cell of the colon and rectum [6]. Aberrant crypt foci (ACF), a colon carcinoma precursor in humans and rats, is selected as one of the feasible tools and as a sensitive, reliable and rapidly appearing biomarker, supported by the presence of histopathologic intraepithelial neoplasia [7]. Pereira et al. [8] evaluated the ability of a substance to reduce the yield of azoxymethane (AOM)-induced foci in the colon of male Fischer 344 rats as a screening assay for 
TABle 1: Herbal composition of ASMq $[4,5]$.

\begin{tabular}{llll}
\hline Plant name & Family name & Uighur name & Used parts \\
\hline Adiantum capillus-veneris L. & Adiantaceae & Pirsiyavxan & Whole plant \\
Alhagi pseudoalhagi Desv. & Fabaceae & Yantak xikiri & Branch secretion \\
Anchusa italica Retz. & Boraginaceae & Gavzivan & Aerial parts \\
Cordia dichotoma G.Forst. & Boraginaceae & Serpistan & Fruit \\
Euphorbia humifusa Willd. & Euphorbiaceae & Xahtere & Whole plant \\
Foeniculum vulgare Mill. & Apiaceae & Arpabidiyan & Fruit \\
Glycyrrhiza uralensis Fisch. & Fabaceae & Qüqük buya yiltizi & Radix or rhizoma \\
Lavandula angustifolia Mill. & Lamiaceae & Üstihuddus & Aerial parts \\
Melissa officinalis L. & Lamiaceae & Badrenjibuya hindi & Whole plant \\
Ziziphus jujuba Mill. & Rhamnaceae & Qilan & Fruit \\
\hline
\end{tabular}

chemopreventive agents, and demonstrated that the ACF might be a useful biomarker to detect possible effects of a chemopreventive agent in rat colon carcinogenesis. In the past several years, a number of chemical carcinogens such as 1,2-dimethylhydrazine (DMH), AOM, 2-amino-3-methyl imidazole quinoline, methylnitrosurea, $N$-methyl- $N$-nitro$N$-nitrosoguanidine have been used to induce benign and malignant neoplasm in the colon of the rodents. These agents have provided a reasonably accurate experimental model of human colon cancer $[9,10]$. One such chemical $\mathrm{DMH}$, a potent and complete carcinogen, has been reliably used to induce initiation and promotion steps of colon carcinogenesis in rodents. DMH and related compounds induce neoplasm specifically in colon of rat even after a single dose $[11,12]$. Metabolic activation of DMH to highly reactive electrophiles (methyldiazonium ion) occurs in the liver and colon. However, the main target organ of DMH is the large intestine [9].

As a number of natural compounds prevent colon carcinogenesis in rodents by inhibition of ACF development [13-21], the present study was designed to determine the potential chemopreventive effects of ASMq ethanol extract against the development of colonic ACF in an experimental model of colon carcinogenesis induced by $\mathrm{DMH}$ treatment in the rats.

\section{Material and Methods}

2.1. Animals. Rats were fed according to ethical standards of animal research committee of the Xinjiang autonomous region. A total of 64 male 6-week-old F344 rats, weighing 180-200 g, were obtained from Harlan (Gannat, France). The animals were kept in polypropylene cages (four animals per cage) on hard wood chips as bedding, and covered with metallic grids in a room maintained at $25 \pm 3^{\circ} \mathrm{C}$, $50 \pm 10 \%$ humidity and with a 12 -h light-dark cycle. They were fed commercial chow (standard powdered diet for rodent maintenance, 9609 Harlan Teklad, Gannat, France) and provided tap water ad libitum.

2.2. Chemicals. DMH was obtained from Fluka SigmaAldrich (Switzerland), and dissolved in $0.9 \% \mathrm{NaCl}$ at a concentration of $2 \mathrm{mg} / \mathrm{mL}$ and adjusted to $\mathrm{pH} 6.8$ with
$1 \mathrm{M} \mathrm{NaOH}$. Formalin solution (10\%, neutral buffered) and methylene blue were obtained from Sigma-Aldrich (Lyon, France). All other chemicals were of reagent grade.

2.3. Preparation of Ethanol Extract of ASMq. ASMq was composed of 10 kinds of herbal medicinal plants as described in Table 1. Plant materials were purchased from Xinjiang Hospital of Traditional Uighur Medicine (Urumqi 830001, P.R. China). Voucher specimens have been deposited in the Herbarium of Xinjiang Institute of Ecology and Geography, Chinese Academy of Sciences (Urumqi 830011, P.R. China). All herbal materials were authenticated from Xinjiang Institute for Drug Control (Urumqi 830002, P.R. China). Herbal medicines were selected according to the usual recipes recommended by Upur and Yusup [5] and prepared by a professional pharmacist. Briefly, herbs were minced and circulated with $95 \%$ ethanol in the proportion of $1: 10(\mathrm{w} / \mathrm{v})$ for $5 \mathrm{~h}$ and repeated for additional three times for $4 \mathrm{~h}$ after adding the same amount of ethanol. The resulting crude extracts were filtered and distilled to oiliness form, and degreased with petroleum ether after dissolving in proper volume of hot water. Remaining water-soluble extract was vacuum dried (Buchi, Switzerland) to powder and kept at $4{ }^{\circ} \mathrm{C}$ for future use. Extract yield of plant material was $12 \%$ $(\mathrm{w} / \mathrm{w})$. In this experiment, the dried powder was dissolved in distilled water as a $1600 \mathrm{mg} / \mathrm{mL}$ (dry weight of the extract) stock solution and could be kept at $-20^{\circ} \mathrm{C}$ for 3 months without loss of any effect. The other concentrations used in the experiment were prepared by adding distilled water weekly.

2.4. Experimental Design. After 1 week of acclimatization, animals were randomly divided into eight groups each containing eight rats (Figure 1): Group 1 was served as control, no DMH injection, treated daily with normal saline at $10 \mathrm{~mL} / \mathrm{kg}$ body weight until the end of the study. Rats in Groups 2-8 were given a single intraperitoneal injection of DMH (20 mg/kg body weight) at the beginning of the study. Rats in Group 2, served as negative control, in addition to DMH injection, were administered normal saline once a day, during the experimental period. Rats in Groups 3-5 served as pretreatment group, ASMq ethanol extract at 400, 800 and $1600 \mathrm{mg} / \mathrm{kg}$ body weight were administered by gavage 
TABLE 2: Effect of ASMq ethanol extract on ACF formation in DMH-treated rats (mean $\pm \mathrm{SD}$ ).

\begin{tabular}{lccc}
\hline \multirow{2}{*}{ Group/treatment } & Number of rats & \multicolumn{2}{c}{ ACF formation in rat colon } \\
Total ACF \\
\hline Normal control (NS) & 8 & Total AC & 0 \\
Negative control (DMH + NS) & 8 & $129.8 \pm 43.7$ & $50.3 \pm 16.8$ \\
Pretreatment (DMH + ASMq 400) & 8 & $105.1 \pm 60.9^{*}$ & $41.9 \pm 20.5^{*}$ \\
Pretreatment (DMH + ASMq 800) & 8 & $91.5 \pm 37.4^{*}$ & $38.8 \pm 14.1^{*}$ \\
Pretreatment (DMH + ASMq 1600) & 8 & $53.4 \pm 31.8^{*}$ & $23.0 \pm 14.0^{*}$ \\
Treatment (DMH + ASMq 400) & 8 & $91.8 \pm 33.6^{*}$ & $35.9 \pm 11.8^{*}$ \\
Treatment (DMH + ASMq 800) & 8 & $81.5 \pm 39.5^{*}$ & $31.0 \pm 14.8^{*}$ \\
Treatment (DMH + ASMq 1600) & 8 & $46.0 \pm 25.8^{*}$ & $22.4 \pm 9.3^{*}$ \\
\hline
\end{tabular}

$* P<.05$ as compared with negative control group.

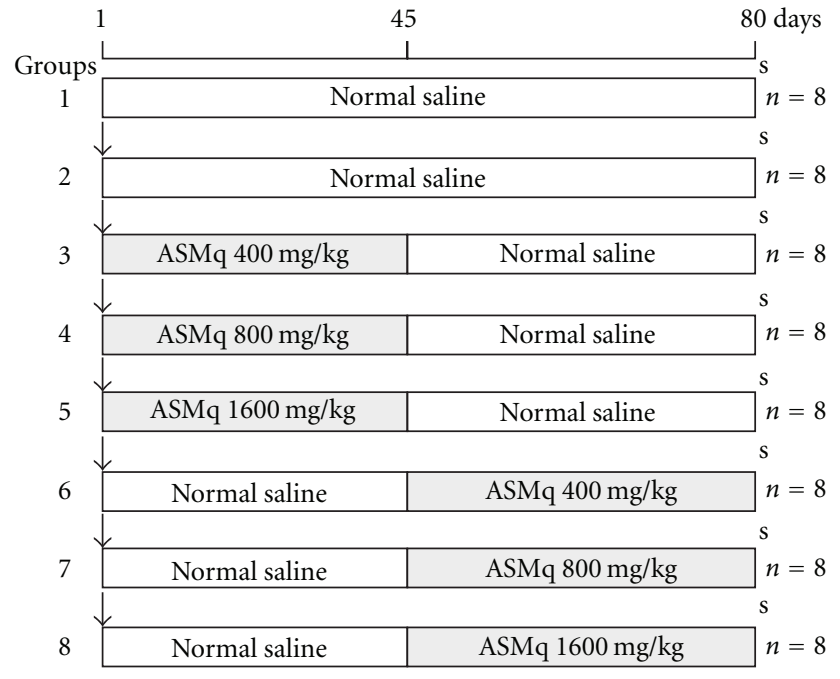

Figure 1: Experimental design to evaluate the effect of ASMq ethanol extract on DMH-induced ACF in the rat colon. Open square: normal saline, yellow square: ASMq administration by gavage, DMH treatment: $20 \mathrm{mg} / \mathrm{kg}$ body weight intraperitoneal injection, s: sacrifice at 91st day, $n$ : number of animals/group, Group 1: normal control, no treatment, Group 2: negative control, DMH alone, Groups 3-5: pretreatment, ASMq at three different doses (400, 800 and $1600 \mathrm{mg} / \mathrm{kg}$, resp.) and Groups 6-8: treatment, ASMq at three different doses (same as pretreatment group).

once a day, respectively, after the $\mathrm{DMH}$ injection until the end of first 45 days, and then continued by normal saline administration for another 45 days. Rats in groups 6, 7 and 8 , served as treatment groups, and administered with normal saline for the first 45 days from the day of DMH injection, subsequently, ASMq ethanol extract at three different doses (400, 800 and $1600 \mathrm{mg} / \mathrm{kg}$ body weight, by gavage) were administered, respectively, until the end of second 45 days. The animals were weighed once a week throughout the experimental period, and the dose volume was adjusted once weekly based on body weight. The administration procedure was completed in 90 days after the DMH injection.

2.5. Tissue Processing, Identification and Quantification of $A C F$. Diets were removed $24 \mathrm{~h}$ prior to sacrifice, and all rats were sacrificed under ether anesthesia at 91st day after the start of the experiment. The colons were removed, cleaned with saline solution $(0.9 \%)$ and slit open longitudinally from cecum to anus. Each colon was cut into proximal, middle and distal portion of equal length, and fixed flat between two pieces of filter paper in $10 \%$ neutral-buffered formalin for at least $24 \mathrm{~h}$. Later, they were stained with 5\% methylene blue for 5-10 $\mathrm{min}$, and they were then placed on a microscopic slide; the mucosal side was observed through light microscope at 40x magnifications. ACF were counted by the method of Bird [22]. Crypts or distinct foci of crypts were counted as an ACF if they displayed at least two of the following characteristics [17]: (i) occupy a greater area than surrounding crypts; (ii) have a thickened epithelial lining; (iii) have elongated or altered shape of luminal opening; and (iv) have an increased pericryptal zone separating the crypt or foci from surrounding crypts. The total number of ACF and the number of crypts/ACF (multiplicity) were scored blindly by a single observer.

2.6. Statistical Analysis. All data were presented as the mean \pm SD. The statistical analysis was accomplished using SPSS 10.0 software package by one-way analysis of variance (ANOVA) and Student's $t$-test. Differences were considered as statistically significant at $P<.05$.

\section{Results}

3.1. Inhibitory Effect of ASMq Ethanol Extracts on Rat Colon $A C F, A C$ Formation. All rats in Groups 2-8 developed colon $\mathrm{ACF}$ and $\mathrm{AC}$ at the end of the experiment. The number of colon ACF and AC per rat of negative control was $50.3 \pm$ 16.8 and $129.8 \pm 43.7$, respectively. Administration of ASMq ethanol extracts either pretreatment or treatment caused a significant inhibition of colon ACF and AC formation in a dose-response relationship when compared with negative control group $(P<.05)$ (Table 2$)$.

3.2. Inhibitory Effect of ASMq Ethanol Extracts on Aberrant Crypt Multiplicity. The number of ACF consisting more than four aberrant crypts per rat of negative control (11 \pm 3.9$)$ was significantly higher than those of pretreatment or treatment groups $(P<.05)$. As far as aberrant crypt multiplicity 
TABLE 3: Effect of ASMq ethanol extract on aberrant crypt multiplicity in DMH-treated rats (mean \pm SD).

\begin{tabular}{lcccc}
\hline Groups/treatment & Number of rats & ACF $\leq 3$ & ACF $\geq 4$ & AC/ACF \\
\hline Normal control (NS) & 8 & 0 & 0 & $11.0 \pm 3.9$ \\
Negative control (DMH + NS) & 8 & $39.3 \pm 14.3$ & $9.0 \pm 7.3^{*}$ & $2.6 \pm 0.2$ \\
Pretreatment (DMH + ASMq 400) & 8 & $32.9 \pm 14.2$ & $3.4 \pm 0.4^{*}$ \\
Pretreatment (DMH + ASMq 800) & 8 & $32.3 \pm 11.2$ & $2.3^{*}$ & $2.3 \pm 0.2^{*}$ \\
Pretreatment (DMH + ASMq 1600) & 8 & $19.6 \pm 12.5^{*}$ & $2.9^{*}$ & $2.3 \pm 0.5^{*}$ \\
Treatment (DMH + ASMq 400) & 8 & $27.8 \pm 9.0^{*}$ & $6.3^{*} \pm 4.1^{*}$ & $2.5 \pm 0.2$ \\
Treatment (DMH + ASMq 800) & 8 & $24.6 \pm 13.3^{*}$ & $2.6 \pm 0.4$ \\
Treatment (DMH + ASMq 1600) & 8 & $19.9 \pm 7.3^{*}$ & $2.5^{*}$ & $2.0 \pm 0.4^{*}$ \\
\hline
\end{tabular}

${ }^{*} P<.05$ as compared with negative control group.

(AC/ACF) was concerned, crypt multiplicity of negative control $(2.6 \pm 0.2)$ was significantly higher than those of all three pretreatment groups $(P<.05)$. Only the highdose treatment group ( $1600 \mathrm{mg} / \mathrm{kg}$ of ASMq ethanol extract) succeeded to decrease the crypt multiplicity in DMH-treated rats $(P<.05)$ (Table 3$)$. A similar tendency was observed for $\mathrm{ACF}$ in less than three aberrant crypts/rats.

\section{Discussion}

Biochemical, genetic and morphologic studies have shown that ACF and colon tumors share similar alterations, further supporting the hypothesis that the ACF are precursors of colorectal cancer [23-25]. A number of natural compounds that inhibit ACF development have been proved to prevent colon cancer in rodents [13-21, 26-28]. Thus, the ACF assay, using the complete colon carcinogen $\mathrm{DMH}$, has been proposed to evaluate chemopreventive agents for colon carcinogenesis in rats [29-31]. The purpose of this study was basically to investigate the effect of ASMq ethanol extract on the rat colon carcinogenesis induced by $\mathrm{DMH}$, as evaluated by ACF development.

Recent studies suggested that in vitro anticancer properties of ASMq ethanol extract and aqueous extract relate to the inhibition of cell proliferation or cell death triggered by either necrosis (LDH leakage) or apoptosis (caspase-3 activation). Several cellular targets such as cellular macromolecules are impacted by the active molecules. Indeed protein, DNA and RNA synthesis are inhibited [32]. Either related or not a caspase-3-dependent apoptosis is induced, which is also characterized by cell cycle arrest in Sub-G1 phase, downregulation of p53, p21 and bcl2 and upregulation of bax-all shown at transcriptional level in the HepG2 cell line of human liver, which still bears a normal regulating capacity of the p53 gene. Concomitantly, the herbal preparation strongly prevents the indirect effects of oxidative stress on the DNA such as formation of oxidized DNA bases ( $8 \mathrm{OH}-\mathrm{dG}$ ) and/or methylated bases such as m5dC. These effects could be regarded as the prevention by ASMq of an epigenetic pathway also leading possibly to cancer $[4,33]$. The active ingredients in ASMq that exerted the anticancer effect in vitro on Hep-G2 cells have not been fully clarified, but the ethyl acetate extract as well as total flavonoids of ASMq have shown a remarkable cytotoxicity and apoptosis-induction potential in this human hepatoma (HepG2) cell line [34].
Our present results showed that the pretreatment as well as the treatment of ASMq ethanol extract suppressed the development of ACF, AC and crypt multiplicity induced by $\mathrm{DMH}$ single injection at all three doses tested. Especially large ACF (four or more) were significantly inhibited after 45 days of pretreatment or treatment with ASMq ethanol extract, since larger ACF (four or more aberrant crypts per focus) have been considered more likely to progress to tumors [35-37]. Our results also showed that the inhibitory effect of ASMq ethanol extract in the treatment group seemed to be more evident than that of pretreatment group. Carcinogenesis is a multistep process, involving initiation, promotion and progression. According to the literature, the increased number of ACF may reflect the initiation step of colorectal carcinogenesis, while the progressive increase in the number of crypts per ACF may correspond to the promotion step of colon tumorigenesis [23, 35, 38-40]. These results indicated that ASMq ethanol extract was able to inhibit the initiation and promotion steps of colon carcinogenesis in rodents.

Other chemopreventive agents with antioxidant properties have been found to inhibit DMH- and AOM-induced colon carcinogenesis and DNA damage in animal model $[13,15,41,42]$. Although the mechanisms involved in the inhibitory effects of ASMq ethanol extract against ACF formation are not clearly understood, it could be explained by its putative antioxidant activity [43] and protective effects to oxidative stress induced DNA and mitochondrial damage $[2,3]$. Since $\mathrm{DMH}$ is an indirect carcinogen, which has to be metabolized to exert its carcinogenic effect, it could be postulated that ASMq ethanol extract has interference on DMH metabolic pathways. Also, the presence of doseresponse relationship observed in the current study could be attributed to the synergistic activity of congeneric or different compounds increase according to the ASMq ethanol extract dose levels.

In summary, the present study demonstrated the inhibitory effect of ASMq ethanol extract on DMH-induced rat colon carcinogenesis. Of course, as an herbal preparation, ASMq ethanol extract contains a variety of compounds such as alkaloids, flavonoids, polysaccharides, terpenes as well as coumarins (Yusup A, et al., unpublished data), rather than suggesting that chemopreventive action of ASMq ethanol extract may be attributed to the combined effects of various constituents rather than to any single component. 
Suppression of mutagen-induced oncogenes expression and cell cycle progression and direct modulation of $\mathrm{DMH}$ metabolism might be associated to the chemopreventive action of ASMq ethanol extract. Further investigations, including studies elucidating the mechanism involved in its antitumorigenic effects, are warranted to fully evaluate active components for its chemopreventive properties.

\section{Acknowledgments}

This study was supported by National Natural Science Foundation of China (no. 30260128) and by a research grant from Science and Technology Department of Xinjiang Uighur Autonomous Region, China (no. 200733146-4).

\section{References}

[1] H. Upur, A. Yusup, A. Umar, and N. Moore, "Uighur traditional medicine syndrome of abnormal Savda in men is associated with oxidative stress, which can be improved by Munziq and Mushil of Abnormal Savda," Therapie, vol. 59, pp. 483-484, 2004.

[2] A. Ablimit, A. Yusup, and H. Upur, "Studies on the protective effects of Munziq and Mushil of abnormal Savda to $\mathrm{OH}$ induced DNA damage," Zhong Yao Yao Li Yu Lin Chuang, vol. 16, pp. 34-36, 2000.

[3] A. Yusup, H. Upur, A. Umar, and N. Moore, "Protective effects of Munziq and Mushil of abnormal Savda to mitochondrial oxidative damage," Fundamental and Clinical Pharmacology, vol. 18, no. 4, pp. 471-476, 2004.

[4] A. Yusup, H. Upur, I. Baudrimont et al., "Cytotoxicity of abnormal Savda Munziq aqueous extract in human hepatoma (HepG2) cells," Fundamental and Clinical Pharmacology, vol. 19, no. 4, pp. 465-472, 2005.

[5] H. Upur and A. Yusup, Theory of Hilit and Mizaj on Uighur Medicine and Modern Research on it, Science and Technology Press, Urumqi, China, 2003.

[6] E. R. Fearon and B. Vogelstein, "A genetic model for colorectal tumorigenesis," Cell, vol. 61, no. 5, pp. 759-767, 1990.

[7] R. P. Bird, "Observation and quantification of aberrant crypts in the murine colon treated with a colon carcinogen: preliminary findings," Cancer Letters, vol. 37, no. 2, pp. 147151, 1987.

[8] M. A. Pereira, L. H. Barnes, V. L. Rassman, G. V. Kelloff, and V. E. Steele, "Use of azoxymethane-induced foci of aberrant crypts in rat colon to identify potential cancer chemopreventive agents," Carcinogenesis, vol. 15, pp. 10491054, 1994.

[9] M. Kamaleeswari, K. Deeptha, M. Sengottuvelan, and N. Nalini, "Effect of dietary caraway (Carum carvi L.) on aberrant crypt foci development, fecal steroids, and intestinal alkaline phosphatase activities in 1,2-dimethylhydrazine-induced colon carcinogenesis," Toxicology and Applied Pharmacology, vol. 214, no. 3, pp. 290-296, 2006.

[10] J. H. Weisburger, "Colon carcinogens: their metabolism and mode of action," Cancer, vol. 28, no. 1, pp. 60-70, 1971.

[11] B. Delage, R. Groubet, V. Pallet, C. Bairras, P. Higueret, and P. Cassand, "Vitamin A prevents high fat diet-induced ACF development and modifies the pattern of expression of peroxisome proliferator and retinoic acid receptor m-RNA," Nutrition and Cancer, vol. 48, no. 1, pp. 28-36, 2004.
[12] B. Delage, C. Bairras, B. Buaud, V. Pallet, and P. Cassand, "A high-fat diet generates alterations in nuclear receptor expression: prevention by vitamin A and links with cyclooxygenase- 2 and beta-catenin," International Journal of Cancer, vol. 116, pp. 839-846, 2005.

[13] V. Manju, V. Balasubramaniyan, and N. Nalini, "Rat colonic lipid peroxidation and antioxidant status: the effects of dietary luteolin on 1,2-dimethylhydrazine challenge," Cellular and Molecular Biology Letters, vol. 10, no. 3, pp. 535-551, 2005.

[14] A. R. Agner, A. P. Bazo, L. R. Ribeiro, and D. M. Salvadori, "DNA damage and aberrant crypt foci as putative biomarkers to evaluate the chemopreventive effect of annatto (Bixa orellana L.) in rat colon carcinogenesis," Mutation Research, vol. 582, pp. 146-154, 2005.

[15] S. W. Barth, C. Fähndrich, A. Bub et al., "Cloudy apple juice decreases DNA damage, hyperproliferation and aberrant crypt foci development in the distal colon of DMH-initiated rats," Carcinogenesis, vol. 26, no. 8, pp. 1414-1421, 2005.

[16] R. H. Dashwood, "Early detection and prevention of colorectal cancer (review)," Oncology Reports, vol. 6, no. 2, pp. 277-281, 1999.

[17] J. H. Exon and E. H. South, "Effects of sphingomyelin on aberrant colonic crypt foci development, colon crypt cell proliferation and immune function in an aging rat tumor model," Food and Chemical Toxicology, vol. 41, pp. 471-476, 2003.

[18] H. U. Gali-Muhtasib, I. H. Younes, J. J. Karchesy, and M. E. ElSabban, "Plant tannins inhibit the induction of aberrant crypt foci and colonic tumors by 1,2-dimethylhydrazine in mice," Nutrition and Cancer, vol. 39, pp. 108-116, 2001.

[19] A. Hagiwara, H. Yoshino, T. Ichihara et al., "Prevention by natural food anthocyanins, purple sweet potato color and red cabbage color, of 2-amino-1-methyl-6-phenylimidazo[4,5b]pyridine (PhIP)-associated colorectal carcinogenesis in rats initiated with 1,2-dimethylhydrazine," Journal of Toxicological Sciences, vol. 27, pp. 57-68, 2002.

[20] S. Ishizuka and T. Kasai, "Inhibitory effect of dietary wheat bran on formation of aberrant crypt foci in rat colon induced by a single injection of 1,2-dimethylhydrazine," Bioscience, Biotechnology, and Biochemistry, vol. 60, pp. 2084-2085, 1996.

[21] T. Kawamori, T. Tanaka, A. Hara, J. Yamahara, and H. Mori, "Modifying effects of naturally occurring products on the development of colonic aberrant crypt foci induced by azoxymethane in F344 rats," Cancer Research, vol. 55, no. 6, pp. 1277-1282, 1995.

[22] R. P. Bird, "Further investigation of the effect of cholic acid on the induction, growth characteristics and stability of aberrant crypt foci in rat colon," Cancer Letters, vol. 88, no. 2, pp. 201209, 1995.

[23] R. P. Bird, "Role of aberrant crypt foci in understanding the pathogenesis of colon cancer," Cancer Letters, vol. 93, no. 1, pp. 55-71, 1995.

[24] R. P. Bird and L. M. Lafave, "Varying effect of dietary lipids and azoxymethane on early stages of colon carcinogenesis: enumeration of aberrant crypt foci and proliferative indices," Cancer Detection and Prevention, vol. 19, pp. 308-315, 1995.

[25] M. C. Dias, A. L. T. Spinardi-Barbisan, M. A. M. Rodrigues, J. L. V. de Camargo, E. Terán, and L. F. Barbisan, "Lack of chemopreventive effects of ginger on colon carcinogenesis induced by 1,2-dimethylhydrazine in rats," Food and Chemical Toxicology, vol. 44, no. 6, pp. 877-884, 2006. 
[26] T. Morioka, M. Suzui, V. Nabandith et al., "The modifying effect of Peucedanum japonicum, a herb in the Ryukyu Islands, on azoxymethane-induced colon preneoplastic lesions in male F344 rats," Cancer Letters, vol. 205, no. 2, pp. 133-141, 2004.

[27] S. Fukushima, H. Wanibuchi, and W. Li, "Inhibition by ginseng of colon carcinogenesis in rats," Journal of Korean Medical Science, vol. 16, pp. S75-S80, 2001.

[28] K. Deeptha, M. Kamaleeswari, M. Sengottuvelan, and N. Nalini, "Dose dependent inhibitory effect of dietary caraway on 1,2-dimethylhydrazine induced colonic aberrant crypt foci and bacterial enzyme activity in rats," Investigational New Drugs, vol. 24, pp. 479-488, 2006.

[29] P. Cassand, S. Maziere, M. Champ, K. Meflah, F. Bornet, and J.-F. Narbonne, "Effects of resistant starch- and vitamin Asupplemented diets on the promotion of precursor lesions of colon cancer in rats," Nutrition and Cancer, vol. 27, no. 1, pp. 53-59, 1997.

[30] K. Uchida, S. Kado, M. Onoue, and K. Tohyama, "Relationship between the nature of mucus and crypt multiplicity in aberrant crypt foci in the rat colon," Japanese Journal of Cancer Research, vol. 88, pp. 807-814, 1997.

[31] D. E. Corpet and S. Taché, "Most effective colon cancer chemopreventive agents in rats: a systematic review of aberrant crypt foci and tumor data, ranked by potency," Nutrition and Cancer, vol. 43, no. 1, pp. 1-21, 2002.

[32] H. Upur, A. Yusup, I. Baudrimont et al., "Inhibition of cell growth and cellular protein, DNA and RNA synthesis in human hepatoma (HepG2) cells by ethanol extract of abnormal Savda Munziq of traditional Uighur medicine," Evidence-Based Complementary and Alternative Medicine. In press.

[33] A. Yusup, H. Upur, T. Kader, I. Baudrimont, and N. Moore, "Mechanism of abnormal Savda Munziq ethanol extract on HepG2 cell apoptosis," Zhong Cheng Yao, vol. 28, pp. 195-8, 2006.

[34] A. Yusup, H. Upur, T. Kadir, I. Baudrimont, and N. Moore, "Effect of abnormal savda Munziq ethyl acetate extract on the proliferation apoptosis and correlative gene expression in human hepatoma (HepG2) cells," Journal of China Pharmaceutical University, vol. 37, no. 2, pp. 161-164, 2006.

[35] X.-M. Zhang, D. Stamp, S. Minkin et al., "Promotion of aberrant crypt foci and cancer in rat colon by thermolyzed protein," Journal of the National Cancer Institute, vol. 84, no. 13, pp. 1026-1030, 1992.

[36] D. P. Belobrajdic, G. H. McIntosh, and J. A. Owens, "Whey proteins protect more than red meat against azoxymethane induced ACF in Wistar rats," Cancer Letters, vol. 198, no. 1, pp. 43-51, 2003.

[37] C.-K. Shih, W. Chiang, and M.-L. Kuo, "Effects of adlay on azoxymethane-induced colon carcinogenesis in rats," Food and Chemical Toxicology, vol. 42, no. 8, pp. 1339-1347, 2004.

[38] A. P. Bazo, M. A. M. Rodrigues, J. M. Sforcin, J. L. V. de Camargo, L. R. Ribeiro, and D. M. F. Salvadori, "Protective action of propolis on the rat colon carcinogenesis," Teratogenesis Carcinogenesis and Mutagenesis, vol. 22, no. 3, pp. 183-194, 2002.

[39] Y. Hirose, T. Kuno, Y. Yamada et al., "Azoxymethane-induced beta-catenin-accumulated crypts in colonic mucosa of rodents as an intermediate biomarker for colon carcinogenesis," Carcinogenesis, vol. 24, no. 1, pp. 107-111, 2003.

[40] C. M. Fenoglio-Preiser and A. Noffsinger, "Aberrant crypt foci: a review," Toxicologic Pathology, vol. 27, no. 6, pp. 632-642, 1999.
[41] M. Sengottuvelan, R. Senthilkumar, and N. Nalini, "Modulatory influence of dietary resveratrol during different phases of 1,2-dimethylhydrazine induced mucosal lipid-peroxidation, antioxidant status and aberrant crypt foci development in rat colon carcinogenesis," Biochimica et Biophysica Acta, vol. 1760, no. 8, pp. 1175-1183, 2006.

[42] V. Manju and N. Nalini, "Chemopreventive potential of luteolin during colon carcinogenesis induced by 1,2dimethylhydrazine," Italian Journal of Biochemistry, vol. 54, no. 3-4, pp. 268-275, 2005.

[43] A. Yusup and H. Upur, "ESR studies on the antioxidant effects of Munziq and Mushil of abnormal Savda," Zhong Yao Xin Yao Yu Lin Chuang Yao Li, vol. 12, pp. 420-422, 2001. 


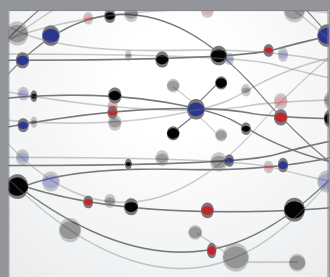

The Scientific World Journal
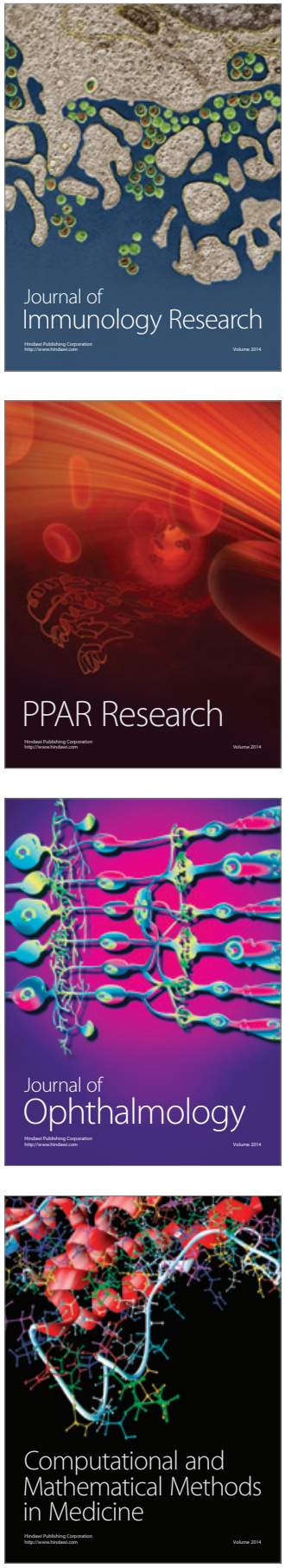

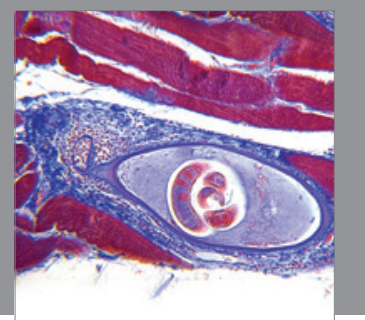

Gastroenterology

Research and Practice
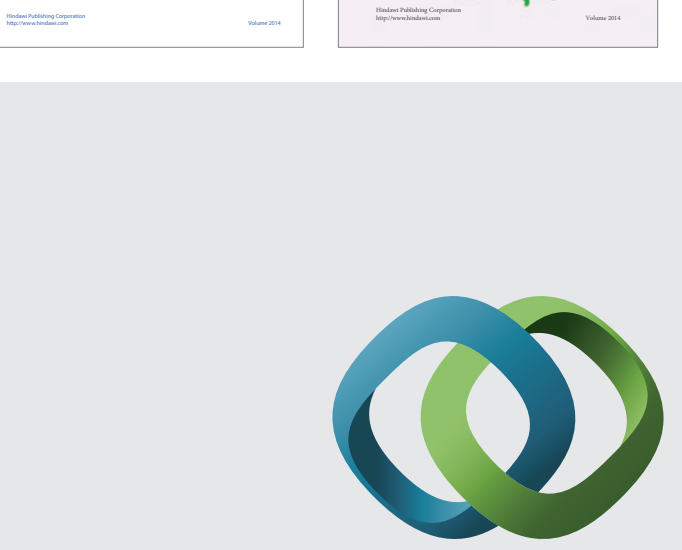

\section{Hindawi}

Submit your manuscripts at

http://www.hindawi.com
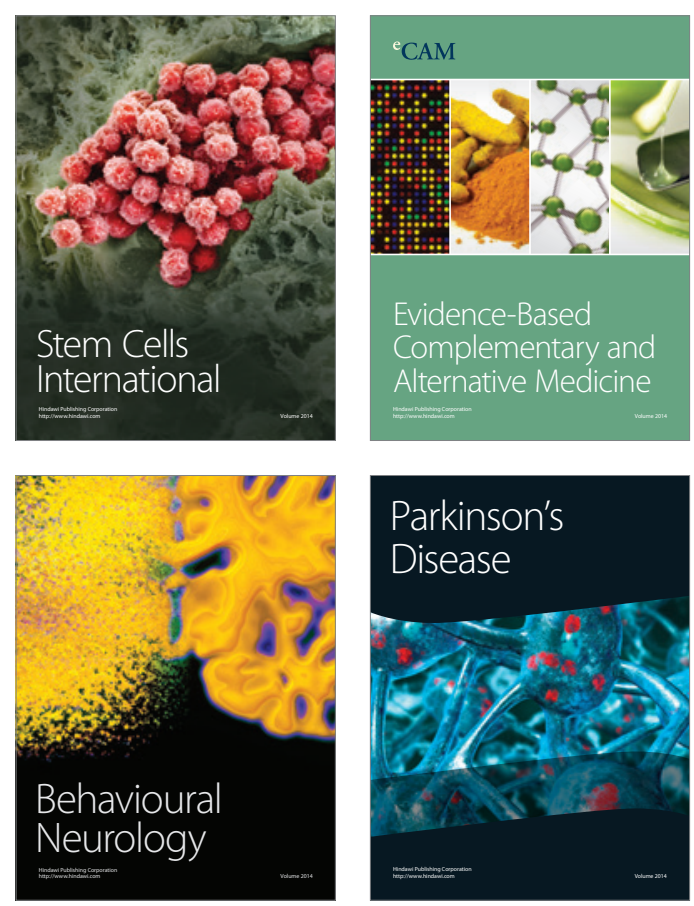

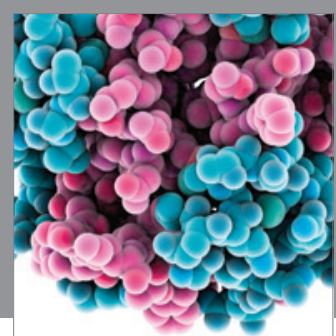

Journal of
Diabetes Research

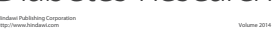

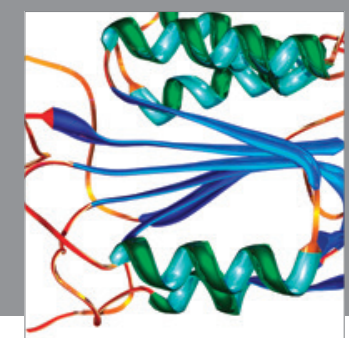

Disease Markers
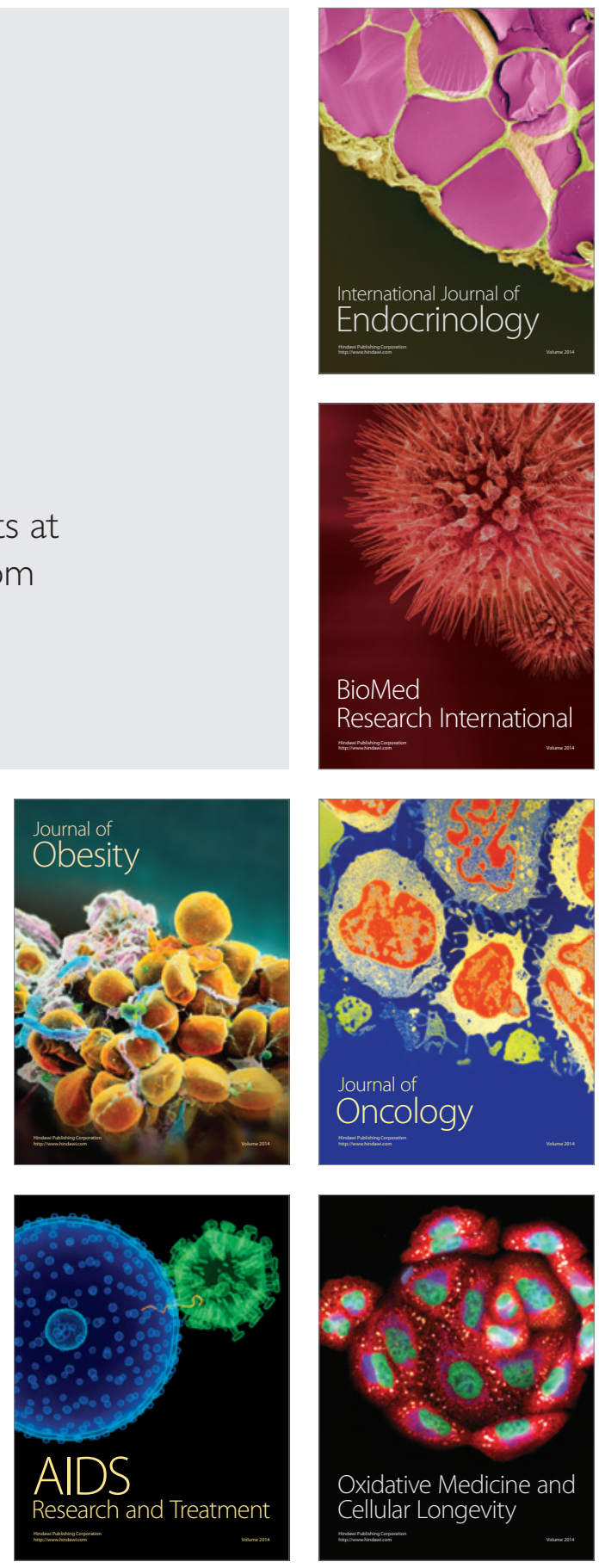